\title{
PQRST: A framework for case discussion and practice-based teaching in general practice training
}

\section{Simon Morgan}

\section{Background}

One of the most important roles of the general practice supervisor is that of clinical teacher. Practice-based teaching comprises formal (structured) and informal (opportunistic) teaching, both of which are primarily based on case discussion. There is no simple framework to guide effective case discussion across both forms of practice-based teaching.

\section{Objective}

In this article, a new five-stage model of case discussion is proposed: PQRST.

The stages are: $P$ - What is the patient's problem?, Q - What is the registrar's question?, R - How well does the registrar reason?, $\mathrm{S}-$ What is the solution?, and T - What can be taught?

\section{Discussion}

The PQRST framework has many benefits. It is a simple framework for case discussion for both formal and informal teaching settings. It emphasises the assessment of clinical reasoning, is time efficient and explicitly prioritises patient and registrar safety. Additionally, it is adaptable for registrars at all levels of training and/or competence.
THE AUSTRALIAN GENERAL PRACTICE TRAINING (AGPT) program is based on the so-called 'apprenticeship model', where accredited general practice supervisors provide clinical oversight to their registrars, who otherwise practice independently. ${ }^{1}$ The general practice supervisor has been defined as 'a general practitioner who establishes and maintains an educational alliance that supports the clinical, educational and personal development of a resident'. ${ }^{2}$ The role of the general practice supervisor is therefore broad and embraces elements of educator, mentor, role model, assessor, coach and pastoral carer. ${ }^{3}$ Arguably, the most prominent aspect of this role is that of teacher.

\section{Teaching in general practice}

Workplace-based teaching in the clinical environment has been defined as 'teaching and learning focused on, and usually directly involving, patients and their problems'. ${ }^{4}$ Teaching in the clinical setting allows direct application of knowledge and skills to patient care and motivates learners to embrace self-directed learning.

General practice is markedly different to other clinical settings, characterised by undifferentiated presentations, comprehensiveness and continuity of care, chronic disease management, processes of care, and the therapeutic influence of the doctor-patient relationship. As a result, teaching in the general practice setting is unique, with a particular focus on patientcentred care, managing uncertainty, clinical reasoning and development of consultation and communication skills.

There are two distinct approaches to general practice-based teaching - formal and informal (Table 1).

\section{Formal teaching}

Formal practice-based teaching is quarantined and structured teaching that is a requirement of both The Royal Australian College of General Practitioners ${ }^{5}$ and The Australian College of Rural and Remote Medicine. ${ }^{6}$ For those registrars training within the AGPT program, it complements the external registrar workshop program delivered by the local regional training organisation (RTO).

There is a wide range of possible teaching methods available for formal practice-based teaching, with case discussion being the most common. ${ }^{7}$

Problem case discussion

In problem case discussion (PCD), the registrar presents their challenging patients to the supervisor and seeks guidance on diagnosis or management. PCD is driven by the complexity of the 
clinical situation as well as the registrar recognising that they need assistance. While meeting the registrar's patient care needs, PCD also allows the supervisor to assess and build on the registrar's clinical knowledge, reasoning skills and tolerance of uncertainty.

There is a lack of a simple framework for PCD in the medical education literature.

\section{Informal teaching}

While formal teaching is regular and structured, informal teaching (also known as corridor or ad hoc teaching) is usually brief and opportunistic. Informal teaching has the potential to be very powerful, as it is driven by, and is responsive to, the registrar's immediate learning needs.

Informal teaching occurs in response to a so-called 'ad hoc supervisory encounter', a hallmark of the apprenticeship model of general practice training.

\section{Ad hoc supervisory encounters}

Ad hoc supervisory encounters are a form of case discussion initiated by the registrar in response to a patient consultation, where the registrar has judged that the situation exceeds their ability to manage independently. ${ }^{8}$ The primary agenda of the ad hoc encounter has been described as enabling the registrar to meet the needs of patient care, but it is also a valuable learning opportunity.

While the content of these opportunistic teaching episodes cannot be planned, the efficacy of informal case discussion and teaching can be improved by using a structured framework. ${ }^{9}$

The 'one-minute preceptor' (OMP) model of clinical teaching, also known as the five-step 'microskills' framework, was developed as a way to assess and care for a patient's needs while also promoting effective learning. ${ }^{10}$ It has been described as a method to jointly diagnose the patient and diagnose the learner's thinking processes.

Another model, WWW-DOC, was developed for managing the ad hoc supervisory encounter when the patient remains present in the room. ${ }^{11}$ Distinctive features of the WWW-DOC model include the supervisor being introduced as providing a 'second opinion' and the use of 'thinking aloud' as a teaching strategy.

A third model of case presentation, SNAPPS, was developed for use in the ambulatory care setting to foster 'a collaborative conversation' between learner and preceptor. ${ }^{12} \mathrm{With}$ its six steps, including narrowing and then analysing the differential, SNAPPS has a specific focus on diagnosis.

\section{The PQRST framework}

In the absence of a simple framework for case discussion and practice-based teaching applicable to both formal PCD and ad hoc supervisory encounters, a new five-stage model has been developed: PQRST (Figure 1 and Box 1). The model was initially developed by the author of this article with reference to his vocational training experience and the international medical education literature. It was then further refined by the team of medical educators responsible for supervisor support and professional development at the author's RTO, GP Synergy.

\section{P - What is the patient's problem?} The PQRST model starts with presentation of the case by the registrar, ideally in a

\section{Table 1. Comparison of formal and informal teaching}

\begin{tabular}{ll}
\hline Formal teaching & Informal teaching \\
\hline - Scheduled & - Opportunistic \\
- Usually 30-60 minutes' duration & $\cdot$ Usually brief (1-5 minutes' duration) \\
- Primary focus on registrar learning & - Primary focus on patient safety \\
- Addresses registrar's less urgent clinical & - Addresses registrar's immediate clinical \\
care and learning needs & care and learning needs \\
\hline
\end{tabular}

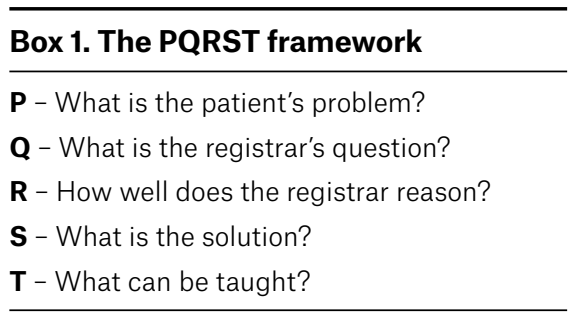

so-called 'problem representation' format. Problem representation has been described as a specific method of case presentation to refine clinical reasoning skills. ${ }^{13}$ It incorporates three key aspects - patient demographics and risk factors, the temporal pattern of illness, and the clinical syndrome.

\section{Q - What is the registrar's question?}

Registrars seek help from their supervisor for a myriad of reasons. Questions may be clinical or non-clinical, trivial or complex, and urgent or non-urgent. The first task of the supervisor after hearing the case presentation is therefore to precisely determine 'What is the registrar's question?' The specific question may not always be clear, and it is important for the supervisor to explicitly clarify this.

Additionally, the supervisor needs to establish the purpose of the registrar's question (ie 'Why is the registrar asking me this question?'). It can be considered that registrars seek help form their supervisors for one of three reasons - 'rescue', assistance or reassurance. It is essential for the general practice supervisor to also understand the reason for the question to respond to it most appropriately.

\section{$\mathbf{R}$ - How well does the registrar reason?} Clinical reasoning has been previously been defined as 'the sum of thinking and decision-making processes associated with practice ... it enables practitioners to take ... the best judged action in a specific context'. ${ }^{14}$ Clinical reasoning encompasses skills in effective data gathering, data synthesis and interpretation, managing uncertainty, shared decision making and evidence-based medicine. It has been described as 'a skill to be learnt rather than a concept to be understood'. ${ }^{15}$

As part of the PQRST framework, the supervisor can effectively assess 


\section{PQRST Model}

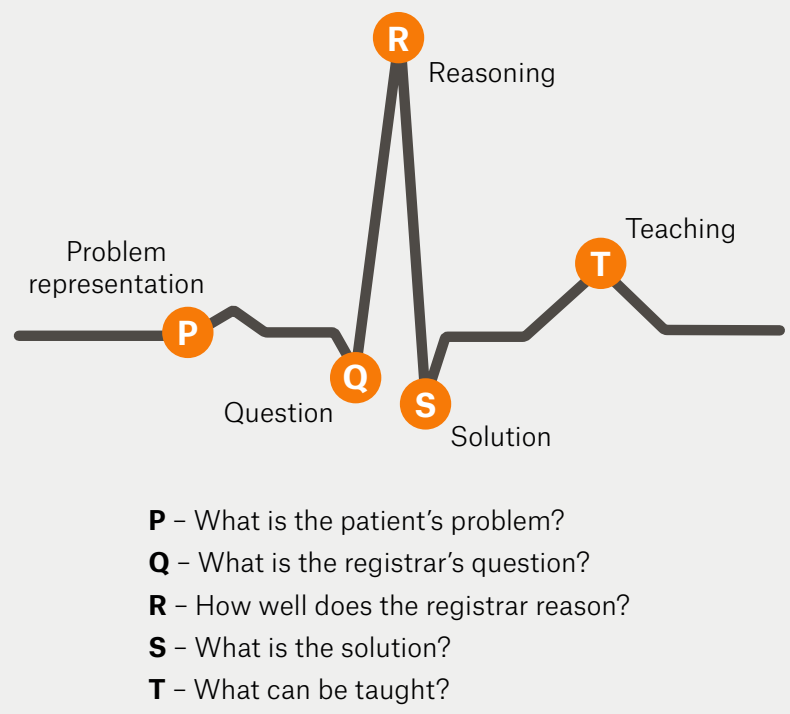

Figure 1. $P Q R S T$ model

their registrar's reasoning skills. This may include comprehensiveness of the problem representation, nature and appropriateness of the probable and differential diagnosis, approach to the undifferentiated presentation, identification of the patient agenda and incorporation of evidence.

The supervisor should probe for additional information on which to base an informed response to the question, and to develop a fuller understanding of the registrar's reasoning skills. Assessment of reasoning skills may be explicit (eg 'So, what features of the presentation make you think of tension headache?') or tacit.

\section{S - What is the solution?}

Registrars seek help from their supervisor for two primary reasons - to maximise the clinical care of the patient, and to address an identified clinical gap or learning need. This can be characterised as enhancing both patient (clinical) safety and registrar (educational and personal) safety. Step four of the PQRST model is therefore for the supervisor to explicitly facilitate a solution to the registrar's question.
While it may be more straightforward for the supervisor to simply give the registrar the answer, and this may be entirely appropriate in some settings (eg struggling registrar, uncomplicated question, time pressures), ideally the supervisor should 'ask before tell'. That is, the supervisor should say something akin to 'So what do you think ...?' before proffering help. This approach has a number of potential advantages - it allows for further assessment of reasoning skills, facilitates deeper learning and helps build registrar confidence.

\section{T - What can be taught?}

Ad hoc supervisory encounters and PCD are primarily based on the need for the registrar to seek an answer to a specific question, but both can and should also be regarded as a potential 'teachable moment'. Unlike PCD, the ad hoc supervisory encounter is brief and opportunistic, and the time to teach may be limited. However, the supervisor can deliver 'micro-teaching' if the environment is appropriate, including pointing the registrar to specific resources or discussing a general approach to the problem.

Box 2 provides an example of the PQRST model.

\section{Discussion}

PQRST is a new framework for case discussion and teaching in the general practice context. This new model has a number of distinct benefits. It meets the need for a simple framework for practicebased case discussion that applies to both the formal PCD and informal ad hoc teaching scenarios. While existing frameworks are potentially adaptable to case discussion in the formal teaching setting, these models were specifically designed for corridor teaching. PQRST is more straightforward for general practice supervisors to apply across both settings, and also to remember as a familiar series of letters from electrocardiogram interpretation.

The explicit identification of the registrar's question makes it more learnercentred, as well as time efficient.

The PQRST model has an overt focus on the assessment and development of a registrar's clinical reasoning skills. Oral case presentations have been described as a valid assessment method to identify a learner's reasoning, in particular in appraising data inclusion, data organisation and the structure and content of the management plan. ${ }^{16}$ The PQRST model addresses all these aspects of clinical reasoning assessment and is unique in having a specific emphasis on problem representation as the initial case presentation structure. ${ }^{13}$

In addition, PQRST explicitly prioritises patient and registrar safety by the specific inclusion of a step for the supervisor to facilitate provision of a solution or answer to the registrar's problem. The OMP, WWW-DOC and SNAPPS models all have a stronger emphasis on teaching and do not directly refer to providing an answer.

The PQRST framework is adaptable to suit registrars at all levels of training and/ or competence. While the clinical urgency of the problem in the ad hoc encounter may limit the scope for teaching, all 


\section{Box 2. Summary example of the PQRST model being used for problem case discussion}

Dan, a first-term general practice registrar, meets his supervisor, Clare, at the end of a busy Thursday to discuss some problem cases.

\section{$\mathbf{P}$ - What is the patient's problem?}

Dan: 'Clare, can I please ask you about a patient I saw a couple of days ago? She is a 38-yearold married schoolteacher with a history of rosacea who presented with gradually worsening pelvic pain over the past two weeks. She denies any other specific symptoms, and there are no red flags such as weight loss or fevers. Pelvic ultrasonography ordered last week shows a simple cyst. She is concerned about ovarian cancer because her auntie was diagnosed with it aged 66 years.'

\section{Q - What is the registrar's question?}

Clare: 'Thanks Dan, so what specifically do you want to ask of me?'

Dan: 'I guess I want to know what more I should do at this stage - that is, should I do more testing or refer her to the gynaecologist?'

\section{$\mathbf{R}$ - How well does the registrar reason?}

Clare: 'Can you tell me what your differential diagnoses are?', 'What are the pros and cons of further imaging?', 'What is the role of tumour markers?', 'What do you think she wants you to do?'

\section{S - What is the solution?}

Clare: 'Before I say what I would do, what do you think is the best approach Dan?'

\section{T - What can be taught?}

Clare: 'Let's talk about ovarian cysts'

the core elements of this model of case discussion - presenting the problem representation, stating the question, assessing reasoning, providing a solution and teaching - are appropriate to all registrars and problems.

The PQRST model has particular utility for new or inexperienced general practice supervisors. It been introduced into both the induction program for new general practice supervisors (the Clinical Teacher Training Workshop) and the 'GP supervisor toolkit' as a case discussion tool at the author's employing RTO, GP Synergy. This organisation administers training for three of Australia's 11 RTOs. However, there has been no formal evaluation of its utility and acceptability in practice. This is an area for future research.

\footnotetext{
Author

Simon Morgan MBBS, FRACGP, MPH\&TM, Medical Educator, GP Synergy, NSW; General Practitioner, Elermore Vale General Practice, NSW

Competing interests: SM is a medical educator working for GP Synergy. He is a member of the Choosing Wisely Advisory Group.

Funding: None.
}

Provenance and peer review: Not commissioned, externally peer reviewed.

Correspondence to:

simon_morgan@gpsynergy.com.au

\section{Acknowledgements}

The author would like to thank Drs Gerard Ingham, Tony Saltis, Sarah Gani and Vanessa Moran for reviewing the manuscript.

\section{References}

1. Trumble SC. The evolution of general practice training in Australia. Med J Aust 2011;194(11):S59-62. doi: 10.5694/j.13265377.2011.tb03129.x.

2. Wearne S, Dornan T, Teunissen PW, Skinner T. General practitioners as supervisors in postgraduate clinical education: An integrative review. Med Educ 2012;46(12):1161-73. doi: 10.1111/j.1365-2923.2012.04348.x.

3. Morgan S. A balancing act: The role of the general practice trainer. Aust Fam Physician 2005;34 Suppl 1;19-22.

4. Spencer J. Learning and teaching in the clinical environment. BMJ 2003;326(7389):591-94. doi: 10.1136/bmj.326.7389.591.

5. The Royal Australian College of General Practitioners. Standards for general practice training 3rd edn. East Melbourne, Vic: RACGP, 2021.

6. Australian College of Rural and Remote Medicine. Primary rural and remote training: Standards for supervisors and teaching posts. Brisbane, Qld: ACRRM, 2013.

7. Ingham G, Morgan S, Kinsman L, Fry J. Are GP supervisors confident they can assess registrar competence and safety, and what methods do they use? Aust Fam Physician 2015;44(4):236-40.

8. Morrison J, Clement T, Nestel D, Brown J. Perceptions of ad hoc supervision encounters in general practice training: A qualitative interview-based study. Aust Fam Physician 2015; 44(12):926-32.

9. Salerno SM, O'Malley PG, Pangaro LN, Wheeler GA, Moores LK, Jackson JL. Faculty development seminars based on the one minute preceptor improve feedback in the ambulatory setting. J Gen Intern Med 2002;17(1):779-87. doi: 10.1046/j.1525-1497.2002.11233.x.

10. Neher JO, Gordon KC, Meyer B, Stevens N. A fivestep 'microskills' model of clinical teaching. J Am Board Fam Pract 1992;5(4):419-24.

11. Ingham G. Avoiding 'consultation interruptus' A model for the daily supervision and teaching of general practice registrars. Aust Fam Physician 2012;41(8):627-29.

12. Wolpaw T, Wolpaw D, Papp K. SNAPPS: A learnercentered model for outpatient education. Acad Med 2003;78(8):893-98. doi: 10.1097/00001888200309000-00010.

13. Audétat MC, Laurin S. Supervision of clinical reasoning: Methods and a tool to support and promote clinical reasoning. Can Fam Physician 2010;56(3):e294-96.

14. Higgs J, Jones MA. Clinical decision-making and multiple problem spaces. In: Higgs J, Jones $\mathrm{M}$, Loftus S, Christensen N, editors. Clinical reasoning in the health professions. 3rd edn. Oxford, UK: Butterworth-Heineman, 2008; p. 3-17.

15. Linn A, Khaw C, Kildea H, Tonkin A. Clinical reasoning. A guide to improving teaching and practice. Aust Fam Physician 2012;41(1-2):18-20.

16. Daniel M, Rencic J, Durning SJ, et al. Clinical reasoning assessment methods: A scoping review and practical guidance. Acad Med 2019;94(6):902-12. doi: 10.1097/ ACM.0000000000002618. 